and rapidly spread upwards. The pulse was rapid and feeble. His temperature had generally been about $100^{\circ} \mathrm{F}$. He died a few hours after he was seen by me, the cause being stated to be asphyxia. I was unfortunately not able to secure a post-mortem examination.

In several of our text-books it is stated that Landry's paralysis is a rapid general motor paralysis, usually starting in the lower extremities without any very marked implica. tion of the sensory apparatus. In looking up the notes of the historical case which occurred in June, 1859, upon which the definition of this disease is in the main based, it is stated that the senses of pain and temperature and the electrical sensibility were not affected, but that the muscular sense was lost in the feet and toes and tactile sensibility was diminished in the distal segments of the limbs and completely lost over the tips of the fingers. Landry states further that "sensibility and motility may be equally affected." Tactile sensibility was very markedly and very generally affected in my case.

I think it highly probable that the disease was the result of influenzal toxæmia. Dr. Powell had no doubt that he was dealing with the ordinary form of influenza for some days antecedent to the onset of the paralysis.

Various other causes are given-e g., exposure to cold and alcoholic excesses. It has been attributed to the poisons of specific fevers, such as small-pox, typhoid, measles, diphtheria, syphilis, and septicæmia. It occurs more often in men than in women, the ratio being three to one. It is a disease chiefly of adult life. The onset may be abrupt or it may be preceded by a variety of premonitory symptoms, mainly of a sensory character. Occasionally they may also be motor or the disease may be ushered in by a feeling of general malaise. The feature which overshadows everything else is the progressive diminution of muscular force, the finer movements disappearing first to be followed by the grosser. Although the feet are generally implicated first, yet this order may be varied by implication of the upper before the lower extremities. In about one-half of the cases the cranial nerves are affected. The nerves most of ten affected are those supplying the muscles of speech and deglutition. In some cases the soft palate alone is, or the soft palate and the epiglottis are, paralysed; in others-and this was a marked feature in my case-the pharynx and the cesophagus are also involved. Facial paralysis has been observed and also paralysis of the extrinsic and intrinsic muscles of the eye. Dr. Judson Bary states that the plantar and other superficial reflexes are probably lost. In my case there was no doubt that these had disappeared. The diaphragm is said to be generally involved before the intercostal muscles. I feel quite sure that this was not so in my case. The actual death-rate I do not know, but it is certainly very high. Dr. Judson Bury states that in 60 per cent. of the fatal cases death occurred before the tenth day; in 40 per cent. it occurred in two, three, or four weeks ; occasionally it is fatal in two days.

The pathology of this disease is at present obscure. In some cases no changes have been noted in the cord or nerves; in others the changes were those usually met with in myelitis; while in a further set neuritis of the peripheral nerves has been the main feature.

Cardiff.

\section{ICHTHYOL AND ITS USES IN SOME SKIN DISEASES.}

\section{BY ALEXANDER BROWNLIE, M.D. EDIN.}

IN publishing notes of some typical cases of eczema and acne treated with ichthyol I would point out that the results have been obtained in private practice, and not in hospitals, where patients have the advantage of trained nurses and resident physicians.

\section{ECZEMA.}

Ichthyol, whether administered internally or applied externally, is, in my opinion, one of the most efficient agents in our hands for treating eczema in all its stages. The cases now quoted to show its value are not taken in any prescribed order but are selected at random from my notebook. The first case which I quote is the first in which I employed ichthyol, and as at that time my knowledge of the substance was limited my notes are naturally fuller of detail than in subsequent cases.

OASE 1.- The patient was a man, aged 33 years, married, with five children. When he was 16 years of age he had severe bæmorrhage during an attack of typhoid fever. $\mathrm{He}$ was of strumous constitution and had suffered from eczema for two years before seeing me in February, 1895. His face and neck were acutely eczematous, weeping, painful, and itchy. On the flexor aspects of his arms and thighs there was a drier form of eczema. His tongue was furred, be was constipated, and he had no appetite. I ordered light diet and gave internally a mixture containing nux vomica, liquor arsenicalis, and chiretta. The following ointment was applied externally twice daily: salicylic acid, five grains ; ichthyol, 30 minims; resorcin, 20 grains; powdered boric acid, 30 grains ; powdered starch, 60 grains; and vaseline to make up two ounces. On Feb. 17th there was no improvement and the itching was very bad; the rintment was also said to cause pain. I therefore stopped it and used the following: subnitrate of bismuth, two drachms; oxide of zinc, one drachm; ichthyol, 30 minims; and vaseline to make up two ounces. On the 21st there was still much itching. On the neck large brown epithelial scales were beginning to peel off. On the 26tb, the arms and legs being dry, I prescribed 20 per cent. ichthyol paint in distilled water, which relieved the itching better tban anything previously tried; 15 grains of trional were also given each night. On March 14th the neck and face showed some improvement, the exudation and pain being less. I now gave two and a half grains of ichthyol in a pill three times a day after food. On the 29th there was a distinct improvement. There was no more exudation and there were no papules on the face. For washing his face the patient used 10 per cent. ichtbyol soap, and the ointment was changed to simple 4 per cent. ichthyol lanoline salve. By the middle of April the arms and legs were distinctly better, but the face and neck still showed some redness and a few papules. In May the patient was sent to Harrogate and be returned greatly improved in general bealth. The only symptoms of eczema now remaining were a slight scaliness of the neck and itching in parts which were quite healed. I therefore sent him to Dr. Allan Jamieson of Edinburgh, who prescribed a carbolic acid and spirit lotion with a weak ichthyol and resorcin ointment. The itching was not relieved at once but it gradually wore off. Any slight recurrence during the next two years was quickly subdued by 5 per cent. ichthyol salve. For the last three years he has had no more attacks.

CASE 2.-This patient was an unmarried man, 26 years of age, who was suffering from eczema of the leg, not varicose. Above the ankle there was a large red itchy patch, showing serous exudation. The treatment was commenced on Feb. 21st, 1895. The part was directed not to be washed with soap but with oatmeal water. Ichthyol in five-grain doses was given internally twice daily, and the following ointment was applied externally: ichthyol, 30 minims; subnitrate of bismuth, two drachms; oxide of zinc, one drachm; powdered starch, two drachms; and vaseline to make up two ounces. On March 1st the patient's condition was much improved. There was now no pain and no weeping, but the part was dry and scaly. A lotion containing 50 minims of ichthyol in an ounce of water was applied twice a day. On the 14th the skin was normal and the patient considered himself to be quite well. He has never been troubled since.

CASE 3.-This patient was a man, 68 years of age. He had a slight tendency to gout, but beyond this there was nothing particular either in his own history or in that of his family. The treatment was commenced on May 5th, 1899. There was eczema of the right leg, which from the knee to the toes was swollen to twice its usual size, pitted on pressure, was everywhere red, inflamed, and weeping, and in many places was covered with crusts, which on being detached left an exuding red surface. On the right hand and arm there were small patches of dry and scaly eczema. Complete rest was ordered with elevation of the leg. A five-grain ichtbyol pill was given thrice daily after food, and a mixture containing stry chnine and digitalis to improve the circulation was ordered. The following ointment was applied twice a day: subnitrate of bismuth, three drachms; ichthyol, 24 minims; and oxide of zinc ointment to make up two ounces. On the 22nd the swelling, pain, and weeping were reduced, and large brown scales were detacbable. The arm and hand were treated with 10 per cent. ichthyol lanolin salve. On June 10th the swelling was almost gone and the leg was in a 
dry and scaly condition, except at a small spot on the back. The following ointment was then applied: ichthyol, one drachm ; ammoniated mercury, 10 grains ; resorcin, 20 grains; vaseline, one ounce; and lanoline to make up two ounces. On the 19 th the leg was normal in size and it was dry. The patient was now allowed to walk. On July 30 th the leg was quite well. This was a bad case, which had already been under other treatment for six monchs. The constant rest was helpful, but the relief afforded by the action of the ichthyol on the dilated vessels was the direct means of cure.

CASE 4.- - This patient was a married woman, 60 years of age, who had for years suffered from recurrent attacks of eczema. Any nervous excitement or worry brought on an attack. Both knees and wrists were scaly, red, and itchy, whilst the lower part of the abdomen and vulva and the upper part of the thigh were red and painful and exuded a copious serous discharge. The treatment was commenced on Oct. 30th, 1899. The abdomen being very painful I applied at first simply a dusting powder of starch and bismuth with a little boric acid. On Nov. 9th I prescribed 15 grains of ichthalbin thrice daily in cachets. On the 17 th there were much less pain and exudation. I now ventured to apply 3 per cent ichthyol lanoline salve which answered admirably; the itching was relieved and the skin soon resumed a normal appearance. On the legs and arms the following ointment was used twice daily: ichthyol, one drachm; ammoniated mercury, 10 grains; oxide of zinc, two drachms; resorcin, 10 grains ; vaseline, six drachms; and lanoline to make up two ounces. An improvement was manifest from the first, and recovery followe $\mathrm{u}$ in six weeks.

In the acute cases no washing with soap was allowed, but with warm oatmeal water instead. When the parts were dry 10 per cent. ichthyol soap was used. No special diet was imposed. Ointments were not merely smeared over, bat were gently rubbed in and the parts wore covered with lint, linen, or silk, no cotton or flannel being allowed to touch the surface. In cases of eczema of the face I have also used with benefit zinc-ichthyol salve muslin, but it is rather diffi sult to keep applied.

\section{ACNE.}

Ichthyol is particularly beneficial in this disease, both in a nne vulgaris and acne rosacea. In the former strong external applications can be borne, but in the latter much weaker strengths must bo used. The best results are obtained when external and internal treatment are combined, and in some cases of acne rosacea in which the skin is too thin and irritable to bear even weak solutions the internal administration of ichthyol alone with steaming will suffice to effect a cure. My general plan of treatment is to begin with five grains of ichthyol thrice daily after food, increasing to 10 grains. Every night and morning the face is steamed for 15 minutes and thin washed with ichthyol soap made into a lather and allowed to dry on, which is then gently washed off with water. After each washing, if it can be borne, ichthyol salve (often combined with ammoniated mercury) is applied. In acne vulgaris, after steaming strong sulphur and ichthyol soap is used, with brisk rubbing by means of a flesh-glove. The diet is regulated. Ichthyol itself relieves mild cases of constipation, but if it does not I give a compound pill of iridin and euonymin or podophyllin.

CASE 1.-This patient, a married woman, 46 years of age, had been treated for acne rosacea for six years. When she was first seen by me in February, 1897, her face was red all over and the smaller blood-vessels were very prominent. The nose, the brow, and the chin were covered with inflamed sebaceous glands, partly papular, others pustular. Her face was painful and she could not go out without a veil. She was placed on five grains of ichthyol thrice daily, and the following ointment was applied twice daily: ichthyol, 25 grains; ammoniated mercury, five grains ; and vaseline and lanoline, of each half an ounce. Improvement was perceptible from the first and in a fortnight it was distinct. The amount of ichthyol was now donbled and extract of nux vomisa was given before meals. In another month there were scarcely any intlamed glands and the redness was confined to a few dilated vessels on the cheeks. Two months later there were no traces of acne and the patient has remained free from it ever since. This case had resisted all remedies for six years; improvement commenced immediately that ichthy ol was given, and to ichthyol I unhesitatingly give the credit of the cure. A similar condition existed in this patient's daughter, aged 24 years; it yielded to ichthyol in an equally satisfactory manner.
CASE 2.- This patient was a female, 17 years of age, who suffered from acne vulgaris. Her face was covered with inflamed sebaceous glands and "blackheads." Constipation was also complained of. Steaming, brisk rubbing with fleshgloves, and the use of sulphur soap were prescribed. In ternally saline purges were given. As this treatment did not do the good that I expected I ordered 10 per cent. ichthyol soap and the following ointment to be rubbed into the skin night and morning: ichthyol, 72 minims ; resorcin, 10 grains; vaseline, two drachms : and lanoline to make up one ounce. Internally five grains of ichthyol were given thrice daily and on every second night a compound iridin pill. Improvement was gradual but sure and in three months the cure was complete.

\section{OTHER APPLICATIONS OF ICHTHYOL.}

I have also used ichthyol with success in many other diseases, such as endometritis, in which $I$ have had some startling results. Although it does not come within the proper scope of this paper I should like to mention one case of endometritis which under the ordinary routine practice would have involved the scraping of the uterine mucous membrane, but which was cured by continued nightly applications of glycerine-ichthyol tampons in the proportion of two drachms of ichthyol to one ounce of glycerine. This patient had had two mucous polypi removed from the cervix without relieving the pain, and she was therefore very reluctant to undergo another operation. 'The ichthyol treatment was persisted in for five months, and ergot, nux vomica, and potassium chlorate were prescribed at the menstrual periods. The result bas been highly satisfactory, as all pain and feeling of "bearing down" are gone and the patient feels quite well again. I had a similar case in an unmarried woman with equally good results.

Recently I have used strong solutions of ichthyol to paint over sprains. The swelling was quickly reduced in the few cases in which I have tried it.

\section{CONCLUSIONS.}

My experience has been that from 2 per cent. to 5 per cent. ichthyol applications are best in acute forms of inflamed skins and from 5 per cent. to 10 per cent strengths in more chronic, drier conditions. In acne vulgaris anything up to $25 \mathrm{per}$ cent.-namely, between 10 per cent. and 25 per cent.--is useful. In all cases the internal administration of the drag is an assistance. I believe that ichthyol taken internally has a direct influence on the skin and is most probably excreted by it. Moreover, the taking of ichthyol internally increases weight, and this applies more particularly to weakly, strumous children.

The one disappointment which I must record is that itch. ing is not relieved as quickly as one might expect, certainly not as quickly as the pain is relieved. Patients express gratitude for the feeling of comfort experienced, but the itching soon returns. 'The itchiness does indeed disappear after a time, but it is always the last symptom to go.

In prurigo and pruritus vulvæ or ani I have not found much benefit to result from the use of ichthyol, particularly in those cases where there is no known organic cause such as diabetes, ulcus cervicis, or anal fissure. I have tried ichthyol in 11 such cases and in only one have I bad satisfactory results. This patient was a pregnant woman with pruritus vulvæ.

When prescribing ointments I always advise the patients to use their oldest under-linen as ichthyol has a staining effect, although it is stated that if quickly washed in warm water any clothing thus stained is made quite clean again.

Ichthyol, of course, is not applicable to every case of eczema or acne, neither do I claim for it a specific action, but it is undonbtedly a useful drug in these diseases.

Redcar.

\section{FOURTEEN AND A HALF HOURS' ARTI- FICIAL RESPIRATION IN A CHILD ONE WEEK OLD; RECOVERY.}

\section{BY GEORGE E. KEITH, M.B., O.M. EDIN.}

TowarDs the end of August I delivered a lady in the country of a healthy boy weighing six and a half pounds. The birth was premature, but only by three weeks. The child was well formed in every respect and, with the exception of a very long and tight foreskin, there was nothing to 